Publ. RIMS. Kyoto Univ. Ser. A

Vol. 3 (1968), pp. 393-416

\title{
On imbedding theorems for Sobolev spaces and some of their generalization
}

By

\author{
Tosinobu Muramatu
}

\section{Introduction}

In the previous paper [9] the auther reports that we can give another proof of imbedding theorems for Sobolev spaces. The purpose of this paper is to develop our proof precisely. We also discuss imbedding theorems for Sobolev spaces with mixed norm and the boundary values of functions belonging to some Sobolev spaces.

For functions $f(x)$ defined in an open set $\Omega$ in the real n-dimensional space $R^{n}$ we introduce the norm

$$
\|f\|_{L^{p, n-m}(\varrho)}=\sup _{2^{\prime \prime} \in \iota^{\prime \prime}}\left\|f\left(x^{\prime}, x^{\prime \prime}\right)\right\|_{L^{p}\left(Q^{\prime}\left(\mathrm{r}^{\prime \prime}\right)\right)},
$$

where $x=\left(x^{\prime}, x^{\prime \prime}\right), x^{\prime} \in R^{m}, x^{\prime \prime} \in R^{n-m}, \Omega^{\prime}\left(x^{\prime \prime}\right)=\left\{x^{\prime} ;\left(x^{\prime}, x^{\prime \prime}\right) \in \Omega\right\}$, and $\Omega^{\prime \prime}$ is the set of all points $x^{\prime \prime}$ such that $\left(x^{\prime}, x^{\prime \prime}\right) \in \Omega$ for some $x^{\prime}$. For $f \in C^{\infty}(\Omega)$ we define the semi-norms ${ }^{1)}$

$$
|f|_{l, p, n-m, \ell}=\sum_{\alpha=l}\left\|D^{\alpha} f\right\|_{L^{p, n-m}(\rho)},
$$

when $l$ is a non-negative integer, or

$$
|f|_{l, p, n-m, \mathcal{Q}}=\sum_{\alpha \mid=[l]} \mid \frac{D^{\alpha} f(x)-D^{\alpha} f(y)}{|x-y|^{l-[l]+m / p}} \|_{L^{p, 2 n-2 m(\rho \times \rho)},}
$$

when $l$ is factional. ${ }^{2}$ We define also the norms

$$
\|u\|_{l, p, n-m, \rho}=\|u\|_{L^{p, n-m}(\rho)}+\mid u_{\mid l, p, n-m, Q}^{\prime} .
$$

Definition 1. The space $W^{l, p, n-m}(\Omega)$ is defined as the completion of the subset of $C^{\infty}(\Omega)$ consisting of functions $f$ with $\|f\|_{l, p, n \rightarrow m, \Omega}<\infty$. $W^{l, p, 0}$ coincides with the usual Sobolev spaces $W^{l, p}$, while $W^{i, p, n}(\Omega)$ coincides with the space $\mathcal{B}^{l}(\Omega)$ of all bounded continuous functions

Received November 1, 1967.

1) $\alpha=\left(\alpha_{1}, \cdots, \alpha_{n 3}\right),|\alpha|=\alpha_{1}+\cdots+\alpha_{n 3} . D^{\alpha}=D_{1}^{\alpha_{1} \cdots} D_{n}^{\alpha_{n}}, D_{j}=\mathrm{\partial} / \partial x_{j}$.

2) $[l]$ denotes the integral part of $l$. 
defined in $\Omega$ whose partial derivatives of order $\leqq l$ all exist and

$$
\sup _{|\alpha|=[l]} \sup _{x, y \equiv 0} \frac{\left|D^{\alpha} f(x)-D^{\alpha} f(y)\right|}{|x-y|^{\mid-[l]}}<\infty
$$

(if $l=[l], D^{\alpha} f$ are continuous and bounded).

In this paper an open set $\Omega$ is said to have the cone property if there exists a bounded uniformly Lipschtz continuous vector-valued function $\Psi(x)$ in $\Omega$ and a constant $T_{0}$ such that for each point $x$ in $\Omega$ the cone

$$
\left\{x+t z+t \Psi(x) ; 0<t \leqq T_{0}, z \in Q\right\} \subset \Omega,
$$

where $Q$ is the unit cube $\left\{z ;\left|z_{j}\right| \leqq 1 / 2\right\}$ in $R^{n}$.

Imbedding theorems are stated as follows.

Theorem 1. Let $\Omega$ be an open set in $R^{n}$ having the cone property. If $1 \leqq p \leqq q<\infty$ and $l-\frac{n}{p} \geqq k-\frac{m}{q}$, then there exists the imbedding:

$$
W^{l, p}(\Omega) \rightarrow W^{k, q, n-m}(\Omega),
$$

with the following exceptional cases.

(a) $1=p, l-n=k-\frac{n}{q}$,

(b) $1<p=q, 0 \leqq m<n, l-\frac{n-m}{p}=k$ is an integer.

It is known that the imbedding also exists when $1<p=q \leqq 2$ and $l-\frac{n-m}{p}=k$ is an integer ([1], [16], [17], [18]). But the author has no proof based on our method.

For $l-\frac{n}{p}>k-\frac{m}{q}$ existence of the imbedding is easily proved by using Hölder's inequality and Jessen's inequality only.

The essential part of the theorem is in the case $l-\frac{n}{p}=k-\frac{m}{q}$.

In the case $0<m<n$ the existence of the imbedding

$$
W^{l, p}(\Omega) \rightarrow W^{k, p, n-m}(\Omega)
$$

means the fact if $f \in W^{l, p}(\Omega)$, then the trace of the function on $m$ dimensional hyperplane $S$ is well defined and belongs to the space $W^{k, q}(\Omega \cap S)$.

Sobolev [14] gave a proof for the case where $l, k$ are integers, 
$m=n$ and $1<p<q$. Sobolev [15] proved for the case where $l$ and $k$ are integers and $l-\frac{n}{p}>k-\frac{m}{q}$. Krondrashov [5] and 'Sobolev [15] discussed also Hölder continuity of the trace (sce $\S 3$. Theorem 1'). Du Plessis [13] proved the case $m=0, k$ is fractional; that is, imbeddings into $\mathcal{B}^{k}(\Omega)$.

Gagliardo [2], [3] and Nirenberg [11] gave a proof for the case $l$ and $k$ are integers, which is quite different from that of Sobolev. They moreover proved "the Gagliardo-Nirenberg inequality",

where

$$
\begin{aligned}
& |u|_{a l, q} \leqq \text { const. }|u|_{l, p}^{a}|u|_{0, r}^{1-a}, \\
& \frac{1}{q}=\frac{a}{p}+\frac{1-a}{r}, \quad 0<a<1 .
\end{aligned}
$$

For the case $l$ or $k$ is fractional, Besov [1], Uspecskii [17], [18] announced their results. Nirenberg [11] also refers to this case. Taibleson [16] also gives a proof for the case $\Omega=R^{n}$.

Another method to investigate the spaces of fractional order is that of the interpolation of spaces. Using this method Peetre [9] gave a proof of the theorem for the spaces of fractional order.

In this paper we employ integrals of the form

$$
\int_{0}^{T} t^{l-1} d t \int K(x, z) f(x+t z+t \Psi(x)) d z
$$

instead of that of potential type. By virtue of the integral representations of the form (1.3) (Lemma 1), after applying Jessen's inequality and Hölder's inequality appropriately, we need only to discuss inequalities concerning integrals of one variable.

\section{\$2. Integral representations and the proof of Theorem 1}

We begin with integral representations:

Lemma 1. Let $\Omega, T_{0}, Q$ and $\Psi(x)$ be as in Theorem 1. Let $\omega(x) \in C^{\infty}\left(R^{n}\right)$ satisfy $\int_{R^{n}} \omega(x) d x=1, \omega(x) \equiv 0$ for $x \notin Q$. Then the following identities hold for any integer $l$ and for any function $f(x) \in C^{\infty}(\Omega)$.

(2.1) $\quad f(x)=\sum_{|\alpha|=l} \int_{0}^{T} t^{l-1} d t \int \frac{(-\Psi(x)-z)^{\alpha}}{\alpha !} f^{(\alpha)}(x+t z+t \Psi(x)) \omega(z) d z^{3)}$

3) $f^{(\alpha)}=D^{x} f$ 


$$
\begin{array}{r}
+\sum_{\mid \alpha<l} \int_{\alpha} \omega_{\alpha}(z)(z+\Psi(x))^{\alpha} f(x+T z+T \Psi(x)) d z, \\
f(x)=\sum_{\mid \alpha=l} \int_{0}^{T} t^{l-1} d t \int \omega_{\alpha}(x, z) f^{(\alpha)}(x+t z+t \Psi(x)) d z \\
+\sum_{\mid \alpha \leqq l} \int \omega_{\alpha}(z)(z+\Psi(x))^{\alpha} f^{(}(x+T z+T \Psi(x)) d z, \\
\left(0<T \leqq T_{0}\right)
\end{array}
$$

where $\omega_{\alpha}(z)$ is a linear combination of derivatives of $\omega$, and

$$
\omega_{\alpha}(x, z)=\frac{(-1)^{l-1}}{\alpha !} \sum_{j=1}^{n} \frac{\partial}{\partial z_{j}}\left\{\left(z_{j}+\Psi_{j}(x)\right)(z+\Psi(x))^{\alpha} \omega(z)\right\} .
$$

For any $\beta,|\beta| \leqq l$,

$$
\begin{aligned}
f^{(\beta)}(x) & =\sum_{\substack{|\alpha|=l \\
\alpha \geq \beta}} \int_{0}^{T} t^{-\mid \beta-1} d t \int \omega_{\alpha-\beta}(x, z) f^{(\alpha)}(x+t z+t \Psi(x)) d z \\
& +T^{-|\beta|} \sum_{|\alpha| \leqq l-\beta_{1}} \int \omega_{\alpha}(z)(z+\Psi(x))^{\alpha} f(x+T z+T \Psi(x)) d z
\end{aligned}
$$

Proof. By Taylor's formula we have

$$
\begin{aligned}
f(x)= & \sum_{|\alpha|=l} \int_{0}^{T} \frac{t^{l-1}}{\alpha !}(-\Psi(x)-z)^{\alpha} f^{(\alpha)}(x+t z+t \Psi(x)) d t \\
& +\sum_{|\alpha|<l} T^{|\alpha|} \frac{(-1)^{|\alpha|}}{\alpha !} f^{(\alpha)}(x+T z+T \Psi(x))(z+\Psi(x))^{\alpha} .
\end{aligned}
$$

Multiplying by $\omega(z)$ and integrating with respect to $z$, we have

$$
\begin{aligned}
f(x) & =\sum_{|\alpha|=l} \int_{0}^{T} t^{l-1} d t \int \frac{l}{\alpha !}(-z-\Psi(x))^{\alpha} f^{(\alpha)}(x+t z+t \Psi(x)) \omega(z) d z \\
& +\sum_{|\alpha|<l} T^{|\alpha|} \frac{(-1)^{|\alpha|}}{\alpha !} \int(z+\Psi(x))^{\alpha} \omega(z) f^{(\alpha)}(x+T z+T \Psi(x)) d z \\
& =f_{1}(x)+f_{2}(x) .
\end{aligned}
$$

From the identity

$$
\begin{aligned}
& \int f^{(\alpha)}(x+T z+T \Psi(x)) g(x, z) d z \\
& =(-T)^{-1 \alpha} \int f(x+T z+T \Psi(x)) D_{z}^{\alpha} g(x, z) d z
\end{aligned}
$$

it follows that

$$
f_{2}(x)=\sum_{\mid \alpha_{\mid}<l} \int \omega_{\alpha}(z)(z+\Psi(x))^{\alpha} f(x+T z+T \Psi(x)) d z .
$$


Using (2.1) with $l$ replaced by $l+1$, we have $f(x)=f_{1}(x)+f_{2}(x)$, where

$$
\begin{aligned}
& f_{1}(x)=\sum_{|\alpha|=l+1} \int_{0}^{T} t^{l} d t \int \frac{l+1}{\alpha !} \omega(z)(-z-\Psi(x))^{\alpha} f^{(\alpha)}(x+t z+t \Psi(x)) d z \\
& f_{2}(x)=\sum_{|\alpha| \leqq l} \int \omega_{\alpha}(x)(z+\Psi(x))^{\alpha} f^{(\alpha)}(x+T z+T \Psi(x)) d z .
\end{aligned}
$$

For each $\alpha$ with $|\alpha|=l+1$ there is an index $j$ such that $D^{\alpha}=D_{j} D^{\beta}$, $|\beta|=l$. Thus,

$$
\begin{aligned}
\int t f^{(\alpha)} & (x+t z+t \Psi(x))(z+\Psi(x))^{\alpha} \omega(z) d z \\
& =\int \frac{\partial}{\partial z_{j}}\left\{f^{(\beta)}(x+t z+t \Psi(x))\right\}(z+\Psi(x))^{\alpha} \omega(z) d z \\
& =-\int f^{(\beta)}(x+t z+t \Psi(x)) \frac{\partial}{\partial z_{j}}\left\{\left(z_{j}+\Psi_{j}(x)\right)(z+\Psi(x))^{\beta} \omega(z) d z .\right.
\end{aligned}
$$

Therefore, $f_{1}(x)$ is equal to the first term on the right side of (2.2). Consider now (2.3). Using (2.2) with $f$ replaced by $f^{(\beta)}$ for $|\beta| \leqq l$, we have

$$
\begin{aligned}
f^{(\beta)}(x) & =\sum_{\substack{\alpha \geqq \beta \\
|\alpha|=l}} \int_{0}^{T} t^{l-1} d t \int \omega_{\alpha-\beta}(x, z) f^{(\alpha)}(x+t z+t \Psi(x)) d z \\
& +\sum_{|\alpha| \leqq l-\mid \beta} \int \omega_{\alpha}(z)(z+\Psi(x))^{\alpha} f^{(\beta)}(x+T z+T \Psi(x)) d z \\
& =f_{1}(x)+f_{2}(x) .
\end{aligned}
$$

Therefore, by (2.5) applied to $\beta$ instead of $\alpha$, we find that $f_{2}(x)$ is equal to the second term on the right-hand side of (2.3). This completes the proof.

Our proof of Theorem is based on these integral representations and the following lemma, which will be proved in the next section.

Lemma 2. Let $\widehat{\Omega}$ be a domain in $R^{n+s}$ having the cone property: there are a constant $T_{0}$ and $\widehat{\Psi}(\hat{x})$ such that $(1.2)$ is satisfied with $\Omega$ and $\Psi$ replaced by $\widehat{\Omega}$ and $\widehat{\Psi}$ respectively. Let $\Omega$ be a domain in $R^{n}$, and let $\Phi$ be a bounded, uniformly Lipschitz continuous mapping from $\Omega$ into $\widehat{\Omega}$ such that $\Phi(x)=\left(x, \Phi_{0}(x)\right)$. Assume that $K(x, \hat{x})$ is a bounded, uniformly Lipschitz continuous function whose carrier is contained 
in $R^{n} \times \widehat{Q}$, where $\widehat{Q}$ is the unit cube in $R^{n+s}$.

For any function $f$ in $L^{p}(\widehat{\Omega})$ we define

$$
V(t, x)=\int K(x, \hat{z}) f(\Phi(x)+t \hat{z}+\widehat{\Psi} \circ \Phi(x)) d z .
$$

(i) Assume that $0 \leqq k<1$ and $1 \leqq p \leqq q<\infty$. Then there is a constant $C$ independent of $f$ and $t$ such that

$$
|V(t, x)|_{k, q, n-m, e} \leqq C t^{-\mu-k}\|f\|_{0, p, \hat{\Omega}}
$$

holds for all $f$ in $L^{p}(\widehat{\Omega})$, where $\mu=\frac{n+s}{p}-\frac{m}{q}$.

(ii) Assume moreover that $l-\mu \geqq k$ and either one of the conditions
(a) $l-\mu>k$,
(b) $1<p<q, m>0$,
(c) $k>0, m=0$, or
(d) $1<p, m<n$

is satisfied. Then there is a constant $C$ independent of $f$ and $T$ such that

$$
\left|\int_{0}^{T} t^{l-1} V(t, x) d t\right|_{k, q, n-m, \varrho} \leqq C T^{l-\mu-k}\|f\|_{0, p, \widehat{\Omega}}
$$

holds for any $f$ in $L^{p}(\widehat{\Omega})$.

Proof of Theorem 1. To prove the theorem, it is enough to show that the inequality

$$
|f|_{k, q, n-m, \varrho} \leqq C\|f\|_{l, p, e}
$$

holds for any $f$ in $W^{l, p}(\Omega) \cap C^{\infty}(\Omega)$, where $C$ is a constant independent of $f$.

Consider first the case in which $l$ is a positive integer. Let $\beta$ be an index with $|\beta|=[k]$. From (2.3) and Lemma 2 it follows that

$$
\left|f^{(\beta)}\right|_{k-[k], q, n-m, \varrho} \leqq C_{1}\left(T^{-\mu^{-k}}|f|_{0, p \Omega}+T^{l-\mu-k}|f|_{l, p, \Omega}\right)
$$

for $f \in W^{i, p}(\Omega) \cap C^{\infty}(\Omega)$, where $\mu=\frac{n}{p}-\frac{m}{q}$. Summing these inequalities over all $\beta$, we have

$$
|f|_{k, q, n-m, \varrho} \leqq C\left(T^{-\mu-k}|f|_{0, p, \Omega}+T^{l-\mu-k}|f|_{l, p, \Omega}\right),
$$

which implies (2.9).

Consider next the case in which $l=[l]+\delta, 0<\delta<1$. Since 


$$
\int \omega_{\alpha-\beta}(x, z) d z=0 \text { and } \int \omega(z) d z=1,
$$

the first term on the right side of (2.3) is equal to

$$
\begin{aligned}
& \sum_{|\alpha|=l, \beta \leqq \alpha} \int_{0}^{T} t^{l-1} d t \iint \omega_{\alpha-\beta}(x, z) \omega(w) \\
& \{f(x+t z+t \Psi(x))-f(x+t w+t \Psi(x))\} d z d w
\end{aligned}
$$

which can be written in the form

$$
\sum_{|\alpha|=l, \beta \leqq \alpha} \int_{0}^{T} t^{l+n \mid p-1} d t \int K_{\alpha-\beta}(x, \hat{z}) f_{\alpha}(\Phi(x)+t \hat{z}+\widehat{\Psi} \circ \Phi(x)) d \hat{z},
$$

where $f_{\alpha}(\hat{x})=\left\{f^{(\alpha)}(x)-f^{(\alpha)}(y)\right\}|x-y|^{-l-n / p}, \Psi(\hat{x})=(\Psi(x), \Psi(y))$ for $\hat{x}=(x, y) \in \Omega \times \Omega, \quad K_{\alpha-\beta}(x, \hat{z})=\omega_{\alpha-\beta}(x, z) \omega(w)|z-w|^{i+n / p}$ for $\hat{z}=(z, w)$ $\in R^{2 n}$, and where $\Phi(x)=(x, x)$. Applying Lemma 2 to these integrals, we have the inequality (2.10), which imply (2.9), and the theorem is proved in this case also.

Taking $q=p, m=n,(2.11)$ implies the first part of the following theorem.

Theorem 2. (The Interpolation Inequality) Let $\Omega$ be as in Theorem 1 and assume that $0<k<l$. Then there is a constant $C$ such that

$$
|f|_{k, p, e} \leqq C\left(\varepsilon^{-k}|f|_{0, p, e}+\varepsilon^{l-k}|f|_{l, p, e}\right)
$$

holds for $f \in W^{i, p}(\Omega)$ and $0<\varepsilon \leqq T_{0}$.

If $\Omega=R^{n}$, then also

$$
|f|_{k, p, R^{n}}=C|f|_{0, p, R^{n}}^{1-k \mid l} \cdot|f|_{l, p, R^{n}}^{k / l}
$$

holds for any $f$ in $W^{l, p}\left(R^{n}\right)$.

Proof. Choosing $\varepsilon=T_{0}=1$, we have for $f \in W^{i, p}\left(R^{n}\right)$

$$
|f|_{k, p} \leqq C\left(|f|_{0, p}+|f|_{l, p}\right) \text {, }
$$

where $C$ denotes a constant depending only on $l, k, p$ and $n$. For $f \in W^{l, p}\left(R^{n}\right)$ set $g(x)=f(\varepsilon x), \varepsilon>0$. It is easily obtained from the definition that

$$
|g|_{k, p}=\varepsilon^{k-n \mid p}|f|_{k, p},
$$

so that (2.14) with $f$ replaced by $g$ implies that 


$$
|f|_{k, p} \leqq C\left(\varepsilon^{-k}|f|_{0, p}+\varepsilon^{l-k}|f|_{l, p}\right) .
$$

Chocsing in (2.15)

$$
\varepsilon=|f|_{0, p}^{1 / l}|f|_{l, p}^{-1 / l}
$$

we have (2.13).

\section{§3. Proof of the fundamental lemma}

We turn now to consider the proof of Lemma 2, which is based on the following lemma.

Lemma 3 . Let $\widehat{\Omega}, T_{0}, \widehat{\Psi}, \Omega$ and $\Phi$ be as in Lemma 2 . For $f \in L^{p}(\widehat{\Omega})$ we define

$$
U(t, x)=\int_{\widehat{Q}}|f(\Phi(x)+t \hat{z}+t \widehat{\Psi} \circ \Phi(x))| d \hat{z} .
$$

(i) If $1 \leqq p \leqq q<\infty$, then there is a constant $C$ independent of $f$ and $t$ such that

where

$$
\begin{aligned}
& \|U(t, x)\|_{0, q, n-m, e} \leqq C t^{-\mu} \| f_{\|, p, \hat{\boldsymbol{\nu}}}, \\
& \mu=\frac{n+s}{p}-\frac{m}{q} .
\end{aligned}
$$

(ii) If $l-\mu>0,1<p<q<\infty$, or if $l-\mu \geqq 0,1<p<q<\infty, m>0$, then there is a constant $C$ independent of $f$ and $T$ such that

$$
\left\|\int_{0}^{T} t^{l-1} U(t, x) d t\right\|_{0, q, n-m, \varrho} \leqq C T^{l-\mu}\|f\|_{0, p, \widehat{Q}} .
$$

(iii) If $l-\mu \geq k>0, m>0,1<p \leqq q<\infty$, and if $p<q$ or $m<n$, then there is a constant $C$ independent of $f$ and $T$ such that for any $x^{\prime \prime}, y^{\prime \prime} \in \Omega^{\prime \prime}$

$$
\begin{array}{r}
{\left[\int_{\ell^{\prime}\left(x^{\prime \prime}\right)} \int_{Q^{\prime}\left(y^{\prime \prime}\right)} \frac{d x^{\prime} d y^{\prime}}{|x-y|^{m+k q}}\left\{\int_{0}^{T} t^{l-1} U(t, x) h\left(\frac{|x-y|}{t}\right) d t\right\}^{q}\right]^{1 / q}} \\
\leqq C T^{l-\mu-k}\|f\|_{0, p, \widehat{Q}}
\end{array}
$$

where $x=\left(x^{\prime}, x^{\prime \prime}\right), y=\left(y^{\prime}, y^{\prime \prime}\right)$, and $h(\tau)=\min \left\{1, \tau^{k+\varepsilon}\right\}, 0<\varepsilon<\frac{m}{q}$.

Proof. Changing the variables of integration, $U$ can be written in the form

$$
U(t, x)=\int_{\hat{Q}_{s}}|f(\Phi(x)+t \hat{z})| d \hat{z}
$$


where $\widehat{Q}_{x}=\widehat{Q}+\widehat{\Psi} \circ \Phi(x)$. Since $\widehat{\Psi} \circ \Phi(x)$ is bounded, there is a constant $b$ such that $\widehat{Q}_{x} \subset b \widehat{Q}$ for all points $x$ in $\Omega$. Thus, defining $f$ to vanish outside $\widehat{\Omega}$, we have

$$
U(t, x) \leqq \int_{\widehat{b \hat{z}}}|f(\Phi(x)+t \hat{z})| d \hat{z}=b^{n+s} \int_{\widehat{Q}}|f(\Phi(x)+t b z)| d z .
$$

Therefore, we may assume that $\widehat{\Omega}=R^{n+s}, \Omega=R^{n}$, and $\widehat{\Psi}=0$.

By Hölder's inequality we have

$$
U(t, x) \leqq\left(\int_{Q}|f(\Phi(x)+t \hat{z})|^{p} d \hat{z}\right)^{1 / p}
$$

Changing the variables of integration, we have

$$
U(t, x) \leqq t^{-(n+s) / p}\|f\|_{0, p, R^{n+s}}
$$

so that (3.2) is proved in the case where $m=0$. To prove (3.2) for $m>0$, let $x=\left(y, y^{\prime}\right), \hat{z}=(z, w)$ and let $\Phi(x)=\left(y, \Phi^{*}(x)\right)$, where $y, z \in R^{m}, y^{\prime} \in R^{n-m}$ and $w, \Phi^{*}(x) \in R^{n+s-m}$. From (3.5) and Fubini's theorem it follows that

$$
\begin{aligned}
\int U\left(t, y, y^{\prime}\right)^{p} d y & \leqq \iint_{Q} \int_{Q^{*}}\left|f\left(y+t z, \Phi^{*}(x)+t w\right)\right|^{p} d y d z d w \\
& =\iiint_{Q^{*}}\left|f\left(y+t z, \Phi^{*}(x)+t w\right)\right|^{p} d z d y d w \\
& \leqq\left.\iiint \int f(u, v)\right|^{p} t^{-n-s+m} d z d u d v, \\
& =t^{-n-s^{+m}}\|f\|_{0, p}^{b},
\end{aligned}
$$

where $Q$ and $Q^{*}$ denote the unit cubes. From this inequality and (3.6) it follows that

$$
\begin{aligned}
\|U(t, x)\|_{0, q, n-m} & \leqq\|U(t, x)\|_{0, p, n-m}^{p / q}\left(\sup _{x} U(t, x)\right)^{1-p / q} \\
& \leqq t^{-\mu}\|f\|_{0, p} .
\end{aligned}
$$

This completes the proof of (i).

Since (3.3) is an immediate consequence of (3.2) if $l-\mu>0$, it is sufficient to prove (3.3) in the case where $l-\mu=0,1<p<q, m>0$. By Jessen's inequality we have

$$
\left\{\int\left(\int_{0}^{T} t^{l-1} U(t, x) d t\right)^{q} d x^{\prime}\right\}^{1 / q}
$$




$$
\leqq \int_{0}^{T} t^{l-1} d t \int_{Q_{1}} d z_{1}\left\{\int\left(\int_{Q^{*}}|f(\Phi(x)+t \hat{z})| d z^{*}\right)^{q} d x^{\prime}\right\}^{1 / q}
$$

where $x=\left(x_{1}, x^{\prime}, x^{\prime \prime}\right), z=\left(z_{1}, z^{*}\right), x_{1}, z_{1} \in R, x^{\prime} \in R^{m-1}, \quad x^{\prime \prime} \in R^{n-m}, \quad z^{*} \in$ $R^{n+s-1}$. From (3.2) with $f$ replaced by $f\left(x_{1}+t z_{1}, \cdot\right)$, it follows that

$$
\left\{\int\left(\int_{Q^{*}}|f(\Phi(x)+t \hat{z})| d z^{*}\right) q d x^{\prime}\right\}^{1 / q} \leqq F\left(x_{1}+t z_{1}\right) t^{-(n+s-1) / p+(m-1) / q}
$$

where $F\left(z_{1}\right)=\left\|f\left(z_{1}, \cdot\right)\right\|_{0, p, R^{n+s-1}}$. Thus we have

$$
\left\{\left(\int_{0}^{T} t^{l-1} U(t, x) d t\right)^{q} d x^{\prime}\right\}^{1 / q} \leqq \int_{0}^{T} t^{1 / p-1^{\prime} q-1} d t \int_{Q_{1}} F\left(x_{1}+t z_{1}\right) d z_{1} .
$$

Therefore, it is sufficient to prove that the inequalities

$$
\left\|\int_{0}^{\infty} t^{1 / p-1 / q-1} d t \int_{-1 / 2}^{1 / 2} \mid f(x+t z) d z\right\|_{0, q} \leqq C\|f\|_{0, p}
$$

holds for any $f$ in $L^{p}(R)$. Set $y=x+t z$ and $w=z$. Then an elementary calculation shows that

$$
\int_{0}^{\infty} t^{1 / p-1^{\prime} q-1} d t \int_{-1 / 2}^{1 / 2}|f(x+t z)| d z=C_{1} \int_{-\infty}^{\infty}|f(y)||x-y|^{1 / p-1 / q-1} d y .
$$

The right side of this relation is a function in $L^{q}$ whose $L^{q}$-norm is dominated by $C\|f\|_{0, p}$, where $C$ denotes a constant depending only on $p$ and $q$. (see [7] p.288). Thus (3.3) is proved.

Proof of (iii). Since $t^{l} \leqq T^{l-\mu-k} t^{\mu+k}$, it is sufficient to prove (3.4) in the case where $l-\mu=k$.

First consider the case in which $n>m, p=q$. By Jessen's inequality and (3.2)

$$
\begin{aligned}
& {\left[\int_{R} \int_{R}{ }_{R} \frac{d x^{\prime} d y^{\prime}}{|x-y|^{m+k p}}\left\{\int_{0}^{\infty} t^{l-1} U(t, x) h\left(\frac{|x-y|}{t}\right) d t\right\}^{\left.p^{-1}\right]^{1 / p}}\right.} \\
& \leqq\left[\int \frac{d v^{\prime}}{|v|^{m^{+k p}}}\left\{\int_{0}^{\infty} \int_{Q_{1}} t^{k+1 / p-1} g\left(x_{n}+t z_{n}\right) h\left(\frac{|v|}{t}\right) d t d z\right\}^{p}\right]^{1 / p},
\end{aligned}
$$

where $g\left(u_{n}\right)$ denotes the $L^{p}$-norm of $f\left(u_{1}, \cdots, u_{n}, \cdots, u_{n+s}\right)$ when considered as a function of $\left(u_{1}, \cdots, u_{n-1}, u_{n+1}, \cdots, u_{n+s}\right)$.

Set

$$
G\left(t, x_{n}\right)=\int_{Q_{1}} g\left(x_{n}+t z_{n}\right) d z_{n}=\frac{1}{t} \int_{-t / 2}^{t / 2} g\left(x_{n}+\tau\right) d \tau .
$$

Since 


$$
\begin{aligned}
& \int_{0}^{\infty} t^{k+1 / p-1} G\left(t, x_{n}\right) h\left(\frac{|v|}{t}\right)|v|^{-m \mid p-k} d t \\
& \quad \leqq \int_{0}^{|v| \mid} t^{k+1 / p-1} G\left(t, x_{n}\right)\left|v^{\prime}\right|^{-m \mid p-k} d t+\int_{|v|}^{\infty} t^{1 / p-\varepsilon-1} G\left(t, x_{n}\right)\left|v^{\prime}\right|^{\varepsilon-m \mid p} d t
\end{aligned}
$$

we have

$$
\begin{aligned}
& {\left[\int \frac{d v^{\prime}}{|v|^{m+k p}}\left\{\int_{0}^{\infty} t^{k+1 / p-1} G\left(t, x_{n}\right) h\left(\frac{|v|}{t}\right) d t\right\}^{p}\right]^{1 / p}} \\
& \quad \leqq C_{2}\left\{\int_{0}^{\infty} r^{-1+k p} d r\left(\int_{0}^{r} t^{k+1 / p-1} G\left(t, x_{n}\right) d t\right)^{p}\right\}^{1 / p} \\
& \quad+C_{2}\left\{\int_{0}^{\infty} r^{-1+\varepsilon p} d r\left(\int_{r}^{\infty} t^{1 / p+\varepsilon-1} G\left(t, x_{n}\right) d t\right)^{p}\right\}^{1 / p} \\
& \leqq C_{2}\left(\frac{1}{k}+\frac{1}{\varepsilon}\right)\left\{\int_{0}^{\infty} G\left(t, x_{n}\right)^{p} d t\right\}^{1 / p} .
\end{aligned}
$$

The last inequality is followed from Hardy's inequality ([7], p. 245):

$$
\begin{aligned}
& \int_{0}^{\infty} t^{p \gamma-p}\left(\int_{0}^{t} \tau^{-\gamma} f(\tau) d \tau\right)^{p} d t \leqq\left(\frac{p}{p-\gamma p-1}\right)^{p} \int_{0}^{\infty} f(t)^{p} d t, \\
& \int_{0}^{\infty} t^{-\gamma p}\left(\int_{0}^{\infty} \tau^{\gamma-1} f(\tau) d \tau\right)^{p} d t \leqq\left(\frac{p}{1-\gamma p}\right)^{p} \int_{0}^{\infty} f(t)^{p} d t .
\end{aligned}
$$

Therefore, what we have to show is that

$$
\int_{0}^{\infty} G\left(t, x_{n}\right)^{p} d t \leqq \text { const. } \int_{-\infty}^{\infty} g(t)^{p} d t .
$$

From the inequality

$$
(a+b)^{p} \leqq 2^{p-1}\left(a^{p}+b^{p}\right), a, b \geqq 0,
$$

and Hardy's inequality, it follows that

$$
\begin{aligned}
& \int_{0}^{\infty} G\left(t, x_{n}\right)^{p} d t \\
& \quad \leqq 2^{p-1} \int_{0}^{\infty}\left\{\frac{1}{t} \int_{0}^{t / 2} g\left(x_{n}+\tau\right) d \tau\right\}^{p} d t+2^{p-1} \int_{0}^{\infty}\left\{\frac{1}{t} \int_{0}^{t / 2} g\left(x_{n}-\tau\right) d \tau\right\}^{p} d t \\
& \quad=\int_{0}^{\infty}\left\{\frac{1}{t} \int_{0}^{t} g\left(x_{n}+\tau\right) d \tau\right\}^{p} d t+\int_{0}^{\infty}\left\{\frac{1}{t} \int_{0}^{t} g\left(x_{n}-\tau\right) d \tau\right\}^{p} d t \\
& \leqq\left(\frac{p}{p-1}\right)^{p}\left\{\int_{0}^{\infty} g\left(x_{n}+t\right)^{p} d t+\int_{0}^{\infty} g\left(x_{n}-t\right)^{p} d t\right\} \\
& \quad=\left(\frac{p}{p-1}\right)^{p} \int_{-\infty}^{\infty} g(t)^{p} d t
\end{aligned}
$$


and this gives (3.4) for $p=q, m<n$.

Consider next the case in which $p<q$. By Jessen's inequality, (3.3) with $l$ replaced by $l-k$, and the inequality

$$
\int_{R} h\left(\frac{|x-y|}{t}\right)^{q} \frac{d y^{\prime}}{|x-y|^{\mid m+k q}} \leqq C_{3} t^{-k q}
$$

we have

$$
\begin{aligned}
& \iint\left\{\int_{0}^{T} t^{l-1} h\left(\frac{|x-y|}{t}\right) U(t, x) d t\right\}^{q} \frac{d x^{\prime} d y^{\prime}}{|x-y|^{m+k q}} \\
& \quad \leqq \int d x^{\prime}\left\{\int_{0}^{T} t^{l-1} U(t, x)\left(\int h\left(\frac{x-y}{t}\right)^{q} \frac{d y^{\prime}}{|x-y|^{m+k q}}\right)^{1 / q} d t\right\}^{q} \\
& \quad \leqq C_{3} \int\left\{\int_{0}^{T} t^{l-k-1} U(t, x) d t\right\}^{q} d x^{\prime} \\
& \quad \leqq C_{4} T^{l-k-\mu}\|f\|_{0, \hat{\imath}, \hat{\imath}} .
\end{aligned}
$$

Therefore, Lemma 3 is established.

Now we are in position to prove Lemma 2. It is obvious that Lemma 2 for $k=0$ follows from Lemma 3. Therefore, it it sufficient to consider the case where $k>0$. From the identity

$$
\begin{aligned}
V(t, y)= & \int K(y, \hat{z}) f(\Phi(y)+t \hat{z}+\widehat{\Psi} \circ \Phi(y)) d \hat{z} \\
= & \int K\left(y, \hat{z}+\frac{\Phi(x)-\Phi(y)}{t}+\widehat{\Psi} \circ \Phi(x)-\widehat{\Psi} \circ \Phi(y)\right) \\
& f(\Phi(x)+t \hat{z}+\widehat{t \Psi} \circ \Phi(x)) d \hat{z}
\end{aligned}
$$

and the inequality

$$
\begin{aligned}
& \mid K(x, \hat{z})-K\left(y, \dot{z}+\frac{\Phi(x)-\Phi(y)}{t}+\widehat{\Psi} \circ \Phi(x)-\widehat{\Psi} \circ \Phi(y) \mid\right. \\
& \leqq C_{1} h\left(\frac{|x-y|}{t}\right),
\end{aligned}
$$

where $h(\tau)=\min \left(1, \tau^{k+\varepsilon}\right), k+\varepsilon \leqq 1$, it follows that

$$
\begin{aligned}
& |V(t, x)-V(t, y)| \\
& \quad \leqq C_{1} h\left(\frac{|x-y|}{t}\right)\left\{\int_{Q \cup Q^{\prime}}|f(\Phi(x)+t \hat{z}+t \widehat{\Psi} \circ \Phi(x))| d \dot{z}\right\},
\end{aligned}
$$

where $Q^{\prime}=Q+t^{-1}(\Phi(y)-\Phi(x))+\widehat{\Psi} \circ \Phi(x)-\widehat{\Psi} \circ \Phi(y)$. It is obvious that 


$$
\int_{Q^{\prime}}|f(\Phi(x)+t \hat{z}+\hat{t \Psi} \circ \Phi(x))| d \hat{z}=\int_{Q}|f(\Phi(y)+t \hat{z}+t \widehat{\Psi} \circ \Phi(y))| d \hat{z}
$$

so that

$$
|V(t, x)-V(t, y)| \leqq C_{1} h\left(\frac{|x-y|}{t}\right)\{U(t, x)+U(t, y)\}
$$

In the case where $m>0$ we choose $\varepsilon$ so small that $0<q \varepsilon<m$. Let $x^{\prime \prime}$ and $y^{\prime \prime}$ be any points in $\Omega^{\prime \prime}$. Set $x=\left(x^{\prime}, x^{\prime \prime}\right)$ and $y=\left(y^{\prime}, y^{\prime \prime}\right)$. From (3.8) it follows that

$$
\iint \frac{U(t, x)^{q}}{|x-y|^{m+k q}} h\left(\frac{|x-y|}{t}\right)^{q} d y^{\prime} d x^{\prime} \leqq C_{2} t^{-k q}|U(t, x)|_{0, q, n-m, u}^{q} .
$$

Combining (3.11), (3.12) and (3.2), we obtain (2.7). From (3.10) and the inequality

$$
(a+b)^{q} \leqq 2^{q-1}\left(a^{q}+b^{q}\right), a, b \geqq 0,
$$

it follows that

$$
\begin{aligned}
& \left|\int_{0}^{T} t^{l-1} V(t, x) d l\right|_{k, q, n-m, Q}^{q} \\
& \quad \leq C_{3} \sup _{x^{\prime \prime}, y^{\prime \prime}}\left[\iint\left\{\int_{0}^{T} t^{l-1} h\left(\frac{|x-y|}{t}\right) U(t, x) d t\right\}^{q} \frac{d x^{\prime} d y^{\prime}}{|x-y|^{m+k q}}\right. \\
& \left.\left.\quad+\iiint \int_{0}^{T} t^{l-1} h\left(\frac{|x-y|}{t}\right) U(t, y) d t\right\}^{q} \frac{d x^{\prime} d y^{\prime}}{|x-y|^{m+k q}}\right]
\end{aligned}
$$

Combining (3.13) and (3.4), we have (2.8) in this case.

Finally, we consider the case in which $m=0$. In this case it is obvious that (2.9) is obtained from (3.9) and (3.2). Using (3.2) and (3.11), we have

$$
\begin{aligned}
\int_{0}^{T} t^{l-1} \mid & V(t, x)-V(t, y) \mid d t \\
& \leqq C_{1} \int_{0}^{T} t^{l-1} h\left(\frac{|x-y|}{t}\right)\{U(t, x)+U(t, y)\} d t \\
& \leqq C_{5}\|f\|_{0, p, \hat{Q}} \int_{0}^{T} t^{l-\mu-1} h\left(\frac{|x-y|}{t}\right) d t \\
& \leqq C_{5} T^{l-\mu-k}\|f\|_{0, p, \hat{Q}} \int_{0}^{\infty} t^{k-1} h\left(\frac{|x-y|}{t}\right) d t
\end{aligned}
$$




$$
\leqq C T^{l-\mu-k}\|f\|_{0, p, \hat{\imath}}|x-y|^{k},
$$

where we have used the fact that

$$
\int_{0}^{\infty} t^{k-1} h\left(\frac{\mid x-y !}{t}\right) d t=\left(\frac{1}{k}+\frac{1}{\varepsilon}\right)|x-y|^{k} .
$$

This establishes (2.8) for $m=0$, and the proof of Lemma 2 is complete.

Lemma $2^{\prime}$. Let $\widehat{\Omega}, \widehat{\Psi}, \Phi, \Omega, T_{0}$ and $V(t, x)$ be as in Lemma 2 . Assume moreover that $\Omega=\Omega^{\prime} \times \Omega^{\prime \prime}$ and $\Omega^{\prime} \subset R^{m}$, and that $0<k=l-\frac{n+s-m}{p}<1$.

Then there exists a constant $C$ independent of $f$ and $T$ such that for any points $x^{\prime \prime}$ and $y^{\prime \prime}$ in $\Omega^{\prime \prime}$

$$
\left\|\int_{0}^{T} t^{l-1}\left\{V\left(t, x^{\prime}, x^{\prime \prime}\right)-V\left(t, x^{\prime}, y^{\prime \prime}\right)\right\} d t\right\|_{0, p, \ell^{\prime}} \leqq C\left|x^{\prime \prime}-y^{\prime \prime}\right|^{k}\|f\|_{0 . p, \hat{a}} .
$$

Proof. From Jessen's inequality, (3.11) and (3.2) it follows that

$$
\begin{aligned}
&\left\|\int_{0}^{T} t^{l-1}\left\{V\left(t, x^{\prime}, x^{\prime \prime}\right)-V\left(t, x^{\prime}, y^{\prime \prime}\right)\right\} d t\right\|_{0, p, \Omega^{\prime}} \\
& \leqq C_{1} \int_{0}^{T} t^{l-1} h\left(\frac{\left|x^{\prime \prime}-y^{\prime \prime}\right|}{t}\right)\left\|U\left(t, x^{\prime}, x^{\prime \prime}\right)+U\left(t, x^{\prime}, y^{\prime \prime}\right)\right\|_{0, p, e^{\prime}} d t \\
& \leqq C_{2} \int_{0}^{T} t^{k-1} h\left(\frac{\left|x^{\prime \prime}-y^{\prime \prime}\right|}{t}\right) d t\|f\|_{0, p, e}, \\
& \leqq C\left|x^{\prime \prime}-y^{\prime \prime}\right|{ }^{k}\|f\|_{0, p, e} .
\end{aligned}
$$

This completes the proof of the lemma.

The same argument as in the proof of Theorem 1 gives the following:

Theorem $\mathbb{1}^{\prime}$. Let $\Omega$ be an open set in $R^{n}$ possessing the cone property, and assume that $\Omega=\Omega^{\prime} \times \Omega^{\prime \prime}$ and $\Omega^{\prime} \subset R^{m}$. If $l-\frac{n-m}{p}=k$ is not an integer, there exists a constant $C$ such that for $f \in W^{l, \phi}(\Omega)$

$$
\sup _{x \prime, y y^{\prime \prime} \in \Omega_{\prime \prime \prime}^{\prime \prime}} \frac{\left\|f\left(x^{\prime}, x^{\prime \prime}\right)-f\left(x^{\prime}, y^{\prime \prime}\right)\right\|_{[k], p, e^{\prime}}}{\left|x^{\prime \prime}-y^{\prime \prime}\right|^{k-[k]}} \leqq C\|f\|_{l, p . \Omega} .
$$




\section{\$4. Sobolev spaces with mixed norm}

In this section we shall introduce generalized Sobolev spaces by using "mixed norms", and discuss the imbedding theorem for those spaces.

Definition 2. Let $\Omega$ be an open set in $R^{\nu}$, and assume that $R^{\nu}=$ $S_{1} \times \cdots \times S_{n}$, where $S_{1}, \cdots, S_{n}$ are linear subspaces in $R^{\nu}$. Set $x=$ $\left(x_{1}, \cdots, x_{n}\right)$, where $x_{1} \in S_{1}, \cdots, x_{n} \in S_{n}$. Then we define the $L^{\left(p_{1}, \cdots m_{m}\right), n-m_{n}}$ ( $\Omega$ )-norm

$$
\begin{aligned}
& \|f\|_{0,\left(p_{1} \cdots, p_{m}\right), n-m, \varrho} \\
& =\sup _{x^{(m)}}\left\{\int_{\Omega^{(m-1)}\left(x^{(m)}\right)}\left(\cdots\left(\int_{\Omega\left(x^{(1)}\right)}|f(x)|^{p_{1}} d x_{1}\right)^{1 / p_{1}} \cdots\right)^{p_{m}} d x_{m}\right\}^{1 / p_{m}},
\end{aligned}
$$

where $x^{(j)}=\left(x_{j+1}, \cdots, x_{n}\right), \Omega^{(j)}$ denotes the set of points $x^{(j)}$ such that $\left(x_{1}, \cdots, x_{j}, x^{(j)}\right) \in \Omega$ for some $x_{1}, \cdots, x_{j}$, and $\Omega^{(i)}\left(x^{(j)}\right)$ denotes the set of points $\left(x_{i+1}, \cdots, x_{j}\right)$ such that $\left(x_{i+1}, \cdots, x_{j}, x^{(j)}\right) \in \Omega^{(i)}$.

The definition of the spaces $W^{l,\left(p_{1}, \cdots, p_{m}\right), n-m}(\Omega)$ is the same as that of the spaces $W^{l, p, n-m}(\Omega)$, except that the $L^{p, n-m}(\Omega)$-norm is replaced by $L^{\left(p_{1}, \cdots, p_{m}\right), n-m}(\Omega)$-norm.

We begin with the following lemma, which is a generalization of Lemma 3.

Lemma 4. Assume that $R^{\nu}=S_{1} \times \cdots \times S_{n}$ and $R^{\nu+\sigma}=\widehat{S}_{1} \times \cdots \times \widehat{S}_{n}$, where $S_{1}, \cdots, S_{n}$ and $\widehat{S}_{1}, \cdots, \widehat{S}_{n}$ are linear subspaces. Let $\widehat{\Omega}$ be an open set in $R^{\nu+\sigma}$, and assume that the cone property (1.2) is satisfied with $\Omega$ and $\Psi$ replaced by $\widehat{\Omega}$ and $\widehat{\Psi}$. For each $j$ let $\mathscr{\Phi}_{j}$ be a bounded, uniformly Lipschitz continuous mapping from $\widehat{S_{j}}$ into $S_{j}$ such that $\Phi_{j}\left(x_{j}\right)$ $=\left(x_{j}, \Phi_{0 j}\left(x_{j}\right)\right)$. Let $\Omega$ be an open set in $R^{v}$ such that $\Phi=\mathscr{\emptyset}_{1} \times \cdots \times \Phi_{n}$ maps $\Omega$ into $\widehat{\Omega}$. For $f(\hat{x}) \in L^{\left(p_{1}, \cdots, p_{n}\right)}(\Omega)$ define

$$
U(t, x)=\int_{\widehat{Q}}|f(\Phi(x)+t \hat{z}+t \Psi \circ \Phi(x))| d \hat{z} .
$$

(i) If $1 \leqq p_{j} \leqq q_{j}<\infty, j=1, \cdots, m$, then there is a constant $C$ independent of $f$ and $t$ such that

$$
\|U(t, x)\|_{0,\left(q_{1}, \cdots, q_{m}\right), n-m, \Omega} \leqq C t^{-\mu}\|f\|_{0,\left(p_{1}, \cdots, p_{n}\right), \hat{a}}
$$


holds for any $0<t \leqq T_{0}$ and $f \in L^{\left(p_{1}, \cdots, p_{n}\right)}(\widehat{\Omega})$, where

$$
\mu=\sum_{j=1}^{n} \frac{\nu_{j}+\sigma_{j}}{p_{j}}-\sum_{j=1}^{m} \frac{\nu_{j}}{q_{j}},
$$

$\nu_{j}+\sigma_{j}=\operatorname{dim}\left(\widehat{S}_{j}\right)$ and $\nu_{j}=\operatorname{dim}\left(S_{j}\right)$.

(ii) If $1 \leqq p_{j} \leqq q_{j}<\infty(j=1, \cdots, m)$, and if either one of the conditions
(a) $l-\mu>0$, or
(b) $l-\mu \geqq 0,1<p_{m}<q_{m}, p_{m+1}, \cdots, p_{n} \leqq p_{m}$

is satisfied, then there is a constant $C$ such that

$$
\begin{gathered}
\left\|\int_{0}^{T} t^{l-1} U(t, x) d t\right\|_{0,\left(q_{2} \cdots, q_{m}\right), n-m, \Omega} \\
\leqq C T^{l-\mu}\|f\|_{0,\left(1 \mathbf{1}, \cdots, p_{n}\right), \widehat{\Omega}}
\end{gathered}
$$

holds for any $0<T \leqq T_{0}$ and $f \in L^{\left(p_{1}, \cdots, p_{n}\right)}(\widehat{\Omega})$.

(iii) If $l-\mu \geqq k, 1 \leqq p_{j} \leqq q_{j}<\infty(j=1, \cdots, m)$, and if either one of the conditions
(a) $l-\mu-k>0$,
(b) $p_{m+1}, \cdots, p_{n} \leqq p_{m}<q_{m}, 1<p_{m}$, or (c) $p_{m+1}, \cdots$, $p_{n} \leqq p_{m}, 0<m<n, 1<p_{m}$

is satisfied, then there is a constant $C$ such that

$$
\begin{gathered}
\left.\left\|\int_{0}^{T} \frac{t^{l-1} U(t, x)}{|x-y|^{k+\beta}} h\left(\frac{|x-y|}{t}\right) d t\right\|_{0,(\zeta 1} \ldots, q_{m}\right), n-m .2 \times \Omega \\
\leqq C T^{l-\mu-k}\|f\|_{0, t, \hat{Q}},
\end{gathered}
$$

where $h(\tau)=\min \left\{1, \tau^{k+\varepsilon}\right\}, 0<\varepsilon<\frac{\nu_{1}}{q_{1}}, \cdots, \frac{\nu_{m}}{q_{m}}$, and $\beta=\sum_{j=1}^{m} \frac{\nu_{j}}{q_{j}}$.

Proof. By the same reasoning as in the proof of Lemma 3 we may assume that $\widehat{\Omega}=R^{\nu+\sigma}, \Omega=R^{\nu}$ and $\Psi=0$.

We shall prove the inequalities

$$
\begin{aligned}
\| U\left(t, x_{1}, \cdots,\right. & \left.x_{m}, x^{(m)}\right) \|_{0,\left(q_{1}, \cdots, q_{s}\right), m-s, s_{1} \times \cdots \times s_{m}} \\
& \leqq t^{-o} \int_{\hat{Q}^{(m)}} F\left(\Phi^{(m)}\left(x^{(m)}\right)+t \hat{z}^{(m)}\right) d \hat{z}^{(m)}
\end{aligned}
$$

and

$$
\left\|\frac{U\left(x_{1}, \cdots, x_{m}, x^{(m)}\right)}{|x-y|^{k-\gamma-\delta}} h\left(\frac{|x-y|}{t}\right)\right\|_{0,\left(q_{1}, \cdots, q_{s}\right), m-s, s_{1} \times \cdots \times s_{m}}
$$




$$
\begin{aligned}
& \leqq C_{1} \frac{t^{-\rho}}{\left|x^{(m)}-y^{(m)}\right|^{k+\gamma+\delta}} \\
& \quad \times \int_{Q^{(m)}} F\left(\Phi^{(m)}\left(x^{(m)}\right)+t \hat{z}^{(m)}\right) d \hat{z}^{(m)} h\left(\frac{\left|x^{(m)}-y^{(m)}\right|}{t}\right),
\end{aligned}
$$

where $x^{(m)}$ and $y^{(m)}$ are any points in $S_{m+1} \times \cdots \times S_{n}, \Phi^{(m)}=\Phi_{m+1} \times \cdots \times \Phi_{n}$,

$$
\rho=\sum_{j=1}^{m} \frac{\nu_{j}+\sigma_{j}}{p_{j}}-\sum_{j=1}^{s} \frac{\nu_{j}}{q_{j}}, \quad \gamma=\sum_{j=1}^{s} \frac{\nu_{j}}{q_{j}}, \varepsilon<\delta,
$$

and

$$
F\left(\hat{z}^{(m)}\right)=\left\|f\left(\hat{z}_{1}, \cdots, \hat{z}_{m}, \hat{z}^{(m)}\right)\right\|_{0,\left(p_{1}, \cdots, p_{m}\right), \hat{s}_{1} \times \cdots \times \widehat{s}_{m}} .
$$

Since the integrals on the right side of (4.6) and (4.7) have the same form as on the right side of (4.2), it is sufficient to prove these inequalities for $m=1$. Consider the case in which $s=1$. By Jessen's inequality and (3.2) we have

$$
\begin{aligned}
& \|U(t, x)\|_{0, q_{1}, R^{\nu+\sigma}\left(x^{(1)}\right)} \\
& \leqq \int_{\widehat{Q}^{(1)}} d \hat{z}^{(1)}\left\|\int_{\widehat{Q}_{1}}|f(\Phi(x)+t \hat{z})| d \hat{z}_{\mathbf{1}}\right\|_{0, q_{1}, K^{\nu+\sigma}\left(\lambda^{(1)}\right)}, \\
& \leqq t^{-\rho} \int_{\widehat{Q}^{1)}} F\left(\Phi^{(1)}\left(x^{(1)}\right)+t \hat{z}^{(1)}\right) d \hat{z}^{(1)},
\end{aligned}
$$

where $\rho=\frac{\nu_{1}+\sigma_{1}}{p_{1}}-\frac{\nu_{1}}{q_{1}}$ and $F\left(\hat{z}^{(1)}\right)=\left\|f\left(\cdot, \hat{z}^{(1)}\right)\right\|_{0, p_{1}, s_{1}}$. An elementary calculation shows that

$$
\begin{gathered}
\left\|\left(\sqrt{a^{2}+|y|^{2}}\right)^{-k-m / a_{m}-\delta} h\left(\frac{\sqrt{a^{2}+|y|^{2}}}{t}\right)\right\|_{0, q, R}{ }^{m} \\
\leqq C_{2} a^{-k-\delta} h\left(\frac{a}{t}\right) .
\end{gathered}
$$

Therefore, we have

$$
\begin{aligned}
& \left\|\frac{U(t, x)}{|x-y|^{k+\gamma+\delta}} h\left(\frac{|x-y|}{t}\right)\right\|_{0, q_{1}, R^{\nu+\sigma}\left(x^{(1)}\right) \times R^{\nu+\sigma}\left(y^{(1)}\right)} \\
& \quad=\|U(t, x)\|_{0, q_{1}, R^{\nu+\sigma}\left(x^{(1)}\right)} \cdot\left\|\frac{h\left(t^{-1} \sqrt{a^{2}+|u|^{2}}\right)}{\left(\sqrt{a^{2}+|u|^{2}}\right)^{k+\gamma^{+\delta}}}\right\|_{0, q_{1}, R^{\nu}} \\
& \leqq C_{2} \frac{t^{-\rho}}{a^{k+\delta}} h\left(\frac{a}{t}\right) \int_{\hat{Q}^{(1)}} F\left(\Phi^{(1)}\left(x^{(1)}\right)+t \hat{z}^{(1)}\right) d \hat{z}^{(1)},
\end{aligned}
$$

where $a=\left|x^{(1)}-y^{(1)}\right|$ and $r=\frac{\nu_{1}}{q_{1}}$. Similarly we obtain the inequalities 
(4.6) (4.7) for $s=0$. Thus we complete the proof of (4.6) and (4.7).

The inequality (4.3) is the same as (4.6) with $m$ and $s$ replaced by $n$ and $m$, respectively.

Now we consider (4.4) and (4.5). From Jessen's inequality and (4.6) ((4.7)) it follows that

$$
\begin{aligned}
& \left\|\int_{0}^{T} t^{l-1} U(t, x) d t\right\|_{0,\left(12 \cdots, q_{m-1}\right), n-m, R\left(x_{m}\right)} \\
& \leqq \int_{0}^{T} t^{l-\rho-1} d t \int_{\hat{Q}_{m}} g\left(\Phi_{m}\left(x_{m}\right)+t \hat{z}_{m}\right) d \hat{z}_{m},
\end{aligned}
$$

where

$$
\rho=\sum_{\substack{j=1 \\ j \neq m}}^{n} \frac{\nu_{j}+\sigma_{j}}{p_{j}}-\sum_{j=1}^{m-1} \frac{\nu_{i}}{\nu_{j}},
$$

and

$$
g\left(\hat{z}_{m}\right)=\left\|f\left(\hat{z}_{1}, \cdots, \hat{z}_{m}, \cdots, \hat{z}_{n}\right)\right\|_{0,\left(p_{1}, \cdots, p_{m-1}, p_{m+1}, \cdots, p_{n}\right), R v+\sigma\left(\hat{z}_{m}\right)} .
$$

$$
\begin{aligned}
& \left\|\int_{0}^{T} \frac{t^{l-1} U(t, x)}{|x-y|^{k+\beta}} h\left(\frac{|x-y|}{t}\right) d t\right\|_{0,\left(q_{1}, \cdots q_{m-1}\right) \cdot n-m, R^{\nu}\left(x_{m}\right) \times R^{\nu}\left(y_{m}\right)} \\
& \leqq C_{1} \int_{0}^{T} \frac{t^{l-\rho-1} d t}{\left|x_{m}-y_{m}\right|^{k+\delta}} h\left(\frac{\left|x_{m}-y_{m}\right|}{t}\right) \int_{\widehat{Q}_{m}} g\left(\Phi_{m}\left(x_{m}\right)+t \hat{z}_{m}\right) d \hat{z}_{m},
\end{aligned}
$$

where $\delta=\frac{\nu_{m}}{q_{m}}$. Applying Lemma 3 (ii) ((iii)) to the function on

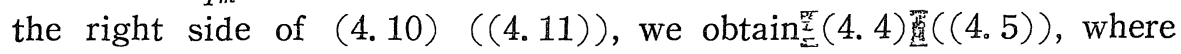
we have used the fact that

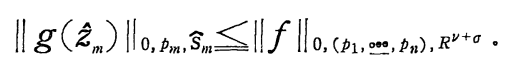

Noting that $p_{m+1}, \cdots, p_{n} \leqq p_{m}$, the above inequality is obtained from Jessen's inequality. Thus the lemma is established.

The following lemma is analogous to Lemma 2.

Lemma 5. Let $\widehat{\Omega}, \Omega, \widehat{\Psi}$ and $\Phi$ be as in Lemma 4 . Let $K(x, \hat{z})$ be a bounded, uniformly Lipschitz continuous function whose carrier is contained in $R^{\nu} \times \widehat{Q}$. For $f \in L^{\left(p_{1}, \cdots, t_{n}\right)}(\Omega)$ define

$$
V(t, x)=\int K(x, \hat{z}) f(\Phi(x)+t \hat{z}+t \widehat{\Psi} \circ \Phi(x)) d \hat{z} .
$$


If $0 \leqq k<1, k \leqq l-\mu, 1 \leqq p_{j} \leqq q_{j}<\infty(j=1, \cdots, m)$, and if either one of the following conditions

(a) $l-\mu>k$,

(b) $m>0, \quad 1<p_{m}<q_{m} ; p_{m+1}, \cdots, p_{n} \leqq p_{m}$,

(c) $n>m>0,1<p_{m} ; p_{m+1}, \cdots, p_{n} \leqq p_{m}, k>0$,

or (d) $k>0, m=0$,

is satisfied, then

$$
\left|\int_{0}^{T} t^{l-1} V(t, x) d x\right|_{k,\left(q_{1}, \cdots, q_{m}\right), n-m, \varrho} \leq C T^{l-k-\mu}\|f\|_{0,\left(p_{1}, \cdots, p_{n}\right), \widehat{Q}}
$$

holds for $0<T \leqq T_{0}$ and $f \in L^{\left(p_{1}, \cdots, p_{n}\right)}(\Omega)$, where $C$ is a constant independent of $f$ and $T$, and $\mu$ denotes the same number as in Lemma 4 .

The proof of this lemma is similar to that of Lemma 2. But we have to use Lemma 4 instead of Lemma 3.

Using the above lemma instead of Lemma 2, by the same argument as in the proof of Theorem 1 we have the following:

Theorem 3. Let $\Omega$ be an open set in $R^{\nu}$ having the cone property, and let $S_{1}, \cdots, S_{n}$ be linear subspaces such that $R^{\nu}=S_{1} \times \cdots \times S_{n}$. If $1 \leqq p_{j} \leqq q_{j}<\infty(j=1, \cdots, m), l-k-\mu \geqq 0$, and if either one of the conditions

(a) $l-k-\mu>0$,

(b) $m>0,1<p_{m}<q_{m} ; p_{m+1}, \cdots, p_{n} \leqq p_{m}$,

(c) $n>m>0,1<p_{m} ; p_{m+1}, \cdots, p_{n} \leqq p_{m}, k$ is not integer,

or (d) $m=0, k$ is not integer,

is satisfied, then there exists the imbedding mapping:

$$
W^{l,\left(p_{1}, \cdots, p_{n}\right)}(\Omega) \rightarrow W^{k,\left(q_{1} \cdots, q_{m}\right), n-m}(\Omega),
$$

where

$$
\mu=\sum_{j=1}^{n} \frac{\nu_{j}}{p_{j}}-\sum_{j=1}^{m} \frac{\nu_{i}}{q .}
$$

and $\nu_{j}=\operatorname{dim} S_{j}$. 


\section{$\S 5$. Boundary values of functions in $\mathbf{W}^{l, p}$}

In this section we discuss boundary values of functions in $W^{l, p}$. First note that we obtain the following three facts by easy arguments;

(a) If $f \in L^{p}\left(R^{n}\right)$ and if for each $\alpha$ with $|\alpha|=[l]$

$$
\frac{D^{\alpha} f(x)-D^{\alpha} f(y)}{|x-y|^{l-[l]+n \mid p}} \in L^{p}\left(R^{n} \times R^{n}\right),
$$

(in the case where $l$ is an integer, $D^{\alpha} f \in L^{p}\left(R^{n}\right)$ )

then $f \in W^{l, p}\left(R^{n}\right)$, where derivatives are taken in the distribution sense.

(b) $C^{s}\left(R^{n}\right) \cap W^{l, p}\left(R_{+}^{n}\right)$ is dense in $W^{l, p}\left(R_{+}^{n}\right)$, where

$$
R_{+}^{n}=\left\{x=\left(x_{1}, \cdots, x_{n}\right), x_{n}>0\right\} \text {, and } l \leqq s .
$$

(c) Let $\Omega$ be a bounded open set in $R^{n}$, and let $\Phi$ be a one-toone $C^{s}$-mapping defined in a neighbourhood of the closure $\bar{\Omega}$ of $\Omega$. Assume that the Jacobian of $\Phi$ does not vanish on $\bar{\Omega}$. If $l \leqq s$, then the correspondence

$$
g \rightarrow f=g \circ \Phi
$$

is a bounded linear transformation from $W^{l, p}(\Phi(\Omega))$ into $W^{i, p}(\Omega)$, and its inverse

$$
f \rightarrow g=f \circ \Phi^{-1}
$$

is also a bounded linear transformation.

We begin with the case $\Omega=R_{+}^{n}$.

Lemma 6. If $1<p<\infty$ and if $l-1 / p=k$ is not integer, then there exists unique bounded linear operator $\gamma$ from $W^{l, p}\left(R_{+}^{\prime \prime}\right)$ into $W^{k, p}\left(R^{n-1}\right)$ such that for $f \in C^{\infty}\left(R^{n}\right) \cap W^{l, p}\left(R_{+}^{n}\right)$

$$
\gamma f\left(x^{\prime}\right)=f\left(x^{\prime}, 0\right), x^{\prime} \in R^{n-1} \text {. }
$$

Proof. The uniqueness follows from the fact (b). It is enough to show the existence of the operator.

From Theorem $1^{\prime}$ it follows that for $f \in C^{\infty}\left(R_{+}^{n}\right) \cap W^{i, p}\left(R_{+}^{n}\right)$

$$
\sup _{x_{n}, y_{n}>0} \frac{\left\|f\left(x^{\prime}, x_{n}\right)-f\left(x^{\prime}, y_{n}\right)\right\|_{[k], p, R^{n-1}}}{\left|x_{n}-y_{n}\right|^{k-[k]}} \leqq C\|f\|_{l, p, R_{+}^{n}}
$$


Therefore, $\left\{f\left(x^{\prime}, \varepsilon\right)\right\}_{\varepsilon>0}$ is a Cauchy sequence in $W^{[k], \phi}\left(R^{n-1}\right)$. Since $W^{[k], p}$ is complete, there exists

$$
\lim _{\varepsilon \rightarrow 0} f\left(x^{\prime}, \varepsilon\right)=g\left(x^{\prime}\right) \text { in } W^{[k], p}\left(R^{n-1}\right) \text {. }
$$

Thus, for each $\alpha=\left(\alpha_{1}, \cdots, \alpha_{n-1}, 0\right)$ with $|\alpha|=[k]$, and for almost every $\left(x^{\prime}, y^{\prime}\right)$ in $R^{n-1} \times R^{n-1}$

$$
\lim _{\varepsilon \rightarrow 0} \frac{D^{\alpha} f\left(x^{\prime}, \varepsilon\right)-D^{\alpha} f\left(y^{\prime}, \varepsilon\right)}{\left|x^{\prime}-y^{\prime}\right|^{\rho}}=\frac{D^{\alpha} g\left(x^{\prime}\right)-D^{\alpha} g\left(y^{\prime}\right)}{\left|x^{\prime}-y^{\prime}\right|^{\rho}},
$$

where $\rho=k-[k]+\frac{n-1}{p}$. Moreover, according to Theorem 1

$$
\sup _{\varepsilon>0}\left\|\frac{D^{\alpha} f\left(x^{\prime}, \varepsilon\right)-D^{\alpha} f\left(y^{\prime}, \varepsilon\right)}{\left|x^{\prime}-y^{\prime}\right|^{\rho}}\right\|_{0, p, R^{n-1} \times R^{n-1}} \leqq C\|f\|_{l, p, R_{+}^{n}},
$$

so that by Fatou's lemma we have

$$
\left\|\frac{D^{\alpha} g\left(x^{\prime}\right)-D^{\alpha} g\left(y^{\prime}\right)}{\left|x^{\prime}-y^{\prime}\right|^{\rho}}\right\|_{0, x, R^{n-1} \times R^{n-1}} \leqq C\|f\|_{l, p, R_{+}^{n}} .
$$

Thus, $g \in W^{k, p}\left(R^{n-1}\right)$, and the correspondence

$$
f \rightarrow g
$$

can be extended uniquely to the bounded linear operator from $W^{l, p}\left(R_{+}^{n}\right)$ into $W^{k, p}\left(R^{n-1}\right)$. The proof is complete.

The above operator is called the trace operator.

We now turn to the general case. Let $\Omega$ be an open set with compact $C^{s}$-boundary $\Gamma$. Then there is a finite open covering $\left\{\boldsymbol{O}_{j}\right\}_{j=1, \cdots, N}$ and $C^{s}$-mappings $\Phi_{j}$ defined in a neighbourhood $O_{j}$ such that $\Phi_{j}$ is oneto-one on $O_{j}$, its Jacobian does not vanish on $\bar{O}_{j}$, and $\Phi\left(\Omega \cap O_{j}\right) \subset R_{+}^{n}$, $\Phi\left(\Gamma \cap O_{j}\right) \subset\left\{x_{n}=0\right\}$. For $\left\{O_{j}\right\}$ we can choose functions $\varphi_{j} \in C_{0}^{\infty}\left(O_{j}\right)$ such that

$0 \leqq \varphi_{j}(x) \leqq 1$, and $\Sigma \varphi_{j}(x)=1$ in a neighbourhoed of $\Gamma$.

Let $l \leqq s$, and let $f \in W^{l, p}(\Omega)$. For each $j \varphi_{j} f \in W^{l, p}\left(\Omega \cap O_{j}\right)$ and $\left\|\varphi_{j} f\right\|_{l, p, \Omega \cap O_{j}} \leqq C_{1}\|f\|_{l, p}$. Set $g_{j}=\varphi_{j} f \circ \Phi_{j}^{-1}$. Since $g_{j}$ is identically zero in a neighbourhood of $\Phi_{j}$ (the boundary of $O_{j}$ ), we may consider that $g_{j} \in W^{l, p}\left(R_{+}^{n}\right)$. From the fact (b) there is a function $g_{j}^{*} \in C^{s}\left(R^{n}\right)$ such that 
Set

$$
\left\|g_{j}-g_{j}^{*}\right\|_{l, p, R+}^{n}<\varepsilon .
$$

$$
f_{j}^{*}(x)= \begin{cases}g_{j}^{*}\left(\emptyset_{j}(x)\right), & x \in O_{j}, \\ 0, & \text { otherwise, }\end{cases}
$$

then $f_{j}^{*} \in C^{s}\left(R^{n}\right) \cap W^{l \cdot p}(\Omega)$, and

$$
\begin{aligned}
\left\|\varphi_{j} f-f_{j}^{*}\right\|_{l, p, \Omega} & \leqq C_{2}\left\|g_{j}-g_{j}^{*}\right\|_{l, p, R+}, \\
& <C_{2} \varepsilon .
\end{aligned}
$$

Since $f_{0}(x)=\left(1-\Sigma \varphi_{j}(x)\right) f(x)$ is identically zero near the boundary, by using the mollifier we find that there is a function $f_{0}^{*}$ in $C^{\infty}\left(R^{n}\right)$ such that

$$
\left\|f_{0}-f_{0}^{*}\right\|_{l, p . \ell}<\varepsilon .
$$

From the above results and the identity

$$
f(x)=f_{0}(x)+\Sigma \varphi_{j}(x) f(x)
$$

it follows that

$$
\left\|f-\left(\sum_{j} f_{j}^{*}+f_{0}^{*}\right)\right\|_{l, p, e}<C_{\varepsilon} .
$$

Therefore, $\boldsymbol{C}^{s}\left(\boldsymbol{R}^{n}\right) \cap \mathbb{W}^{l, p}(\boldsymbol{\Omega})$ is dense in $\mathbb{W}^{l, p}(\boldsymbol{\Omega})$.

Now assume that $l-\frac{1}{p}=k$ is not an integer and let $r_{0}$ be the trace operator from $W^{l, p}\left(R_{+}^{n}\right)$ into $W^{k, p}\left(R^{n-1}\right)$. For $f \in W^{l, p}(\Omega)$ set

$$
\gamma f=\sum_{j}\left\{r_{0}\left(\varphi_{j} f \circ \bar{\Phi}_{j}^{-1}\right)\right\} \circ \Phi_{j} .
$$

Since all the operators

$$
\begin{aligned}
& f \rightarrow \varphi_{j} f, \\
& f \rightarrow f \circ \Phi_{j}^{-1}, \\
& g \rightarrow \gamma_{0} g, \\
& g \rightarrow g \circ \Phi_{j}
\end{aligned}
$$

and

are continuous and linear, $\gamma$ is a continuous linear operator from $W^{l, p}(\Omega)$ into $W^{k, p}(\Gamma){ }^{3)}$ On the other hand, if $f \in C^{s}\left(R^{n}\right) \cap W^{l, p}(\Omega)$, then

3) Let $M$ be an $n$-dimensional compact $C^{s}$-manifold. We say $f \in W^{l, p}(M)$ if $\varphi f \circ \Phi \in$ $W^{l, p}\left(R^{n}\right)$ for every $C^{s}$-coordinate system $\Phi$ and every $C^{s}$-function $\varphi$ whose carrier lies in the domain of $\Phi$. 


$$
g_{j}=\varphi_{j} f \circ \Phi_{j}^{-1} \in C^{s}\left(R^{n}\right)
$$

and

$$
\gamma_{0} g_{j}\left(x^{\prime}\right)=g_{j}\left(x^{\prime}, 0\right)
$$

so that for $x \in \Gamma$

$$
\begin{aligned}
\gamma f & (x)=\sum_{j} \gamma_{0} g_{j}\left(\Phi_{j}(x)\right) \\
& =\sum_{j} g_{j}\left(\Phi_{j}(x)\right) \\
& =\sum \varphi_{j}(x) f(x) \\
& =f(x)
\end{aligned}
$$

Therefore, we have

Theorem 4. Let $\Omega$ be an open set with compact $C^{s}$-boundary $\Gamma$. If $l \leqq s$, and if $l-\frac{1}{p}=k$ is not an integer, then there exists unique continuous linear operator $\gamma$ from $W^{l, p}(\Omega)$ into $W^{k, p}(\Gamma)$ such that for $f \in C^{s}\left(R^{n}\right) \cap W^{l, p}(\Omega)$

$$
\gamma f(x)=f(x), \text { on } \Gamma .
$$

Proof. The uniqueness follows from the fact that $C^{s}\left(R^{n}\right) \cap W^{l, p}(\Omega)$ is dence in $W^{l, p}(\Omega)$.

\section{REFERENCES}

[1] Besov, O.V., On a certain family of functional spaces, imbedding and continuation, Dokl. Akad. Nauk SSSR, 126 (1956), 1163-1165. (Russian)

[2] Gagliardo, E., Proprietà di alcune classi di funzioni in più variabili, Ricerche Mat. 7 (1958), 102-137.

[3] Ulteriori proprietà di alcune classi di funzioni in più variabili, Ricerche Mat. 8 (1959), 24-51.

[4] Gudiev, A. H., An imbedding theorem for restrictions as abstract functions, Dokl. Akad. Nauk SSSR, 147 (1962), 764-767. (Russian)

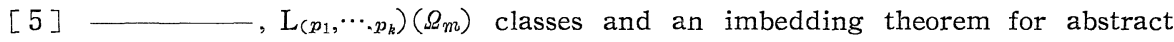
set functions, Dokl. Akad. Nauk SSSR, 149 (1963), 241-244. (Russian)

[6] The Sobolev-Nikol'skii problem for limit exponent, Dokl. Akad. Nauk SSSR, 149 (1963), 509-512. (Russian)

[ 7 ] Hardy, G., J. E. Littlewood and G. Polya, Inequalities, Cambridge University Press, 1934.

[8] Kondrashov, L., On some properties of functions of the spaces $L_{p}$. Dokl. Akad. Nauk SSSR, 148 (1945), 563-566. (Russian)

[9] Muramatu, T., A proof for the imbedding theorems for Sobolev spaces, Proc. Japan Acad. 32 (1967), 87-92.

[10] Nikol' skii, S. M., On imbedding, continuation and approximation theorems for 
differential functions of several variables, Uspehi Mat. Nauk 16, No.5 (1961). 63-114 (Russian); Russian Math. Surveys, 16. No. 5 (1961). 55-104.

[11] Nirenberg, L.. On elliptic partial differential equations. Ann. Scuola Norm. Sup. Pisa, 13 (1959), 115-161.

[12] Peetre, J., Espaces d'interpolation et théorème de Soboleff. Ann. Inst. Fourier, 16 (1966), 279-317.

[13] du Plessis. N., Some theorems about the Riesz fractional integral, Trans. Amer. Math. Soc. 80 (1955), 124-134.

[14] Sobolev, S. L.. On a theorem of functional analysis Mat. Sb. 4 (46) (1938), 471-497. (Russian)

[15] Some applications of functional analysis to mathematical physics, Leningrad, 1950. (Russian) (=Amer. Math. Soc.. Providence. 1963)

[16] Taibleson, M.H.. On the theory of Lipschitz spaces of distributions on Euclidean n-space, I: Principal properties. J. Math. Mech. 13 (1964), 407-479.

[17] Uspenskii, S. V., Imbedding theorem for classes of S. L. Sobolev of fractional order, Dokl. Akad. Nauk SSSR, 130 (1960), 992-993. (Russian)

[18] - Properties of classes $W_{p}^{(\prime)}$ with fractional derivative on differentiable manifolds, Dokl. Akad. Nauk SSSR, 132 (1961), 60-62. (Russian) 